\title{
Erratum: Accuracy of microRNAs as markers for the detection of neck lymph node metastases in patients with head and neck squamous cell carcinoma
}

\author{
Ana Carolina de Carvalho ${ }^{1,3}$, Cristovam Scapulatempo-Neto ${ }^{2}$, Danielle Calheiros Campelo Maia', \\ Adriane Feijó Evangelista ${ }^{3}$, Mariana Andozia Morini ${ }^{2}$, André Lopes Carvalho ${ }^{4,7^{*}}$ and André Luiz Vettore $5,6,8^{*}$
}

\section{Author's correction note}

Upon reviewing our recently published research article [1], we noticed that some of the data presented in the manuscript and on the tables were incorrectly presented. These mistakes do not change the overall conclusions regarding the high accuracy of miR-203 and miR-205 as diagnostic markers of neck metastases in HNSCC.

\section{Corrected text}

1. (Page 8: End of "Validation and diagnostic accuracy of miR-203 and miR-205 expression in FNA samples")

Please replace

All in all, the sensitivity rate for both markers was $92.9 \%(39 / 42$, CI $95 \%, 80.5-98.4)$, with a specificity level of $100 \%$ (71/71, CI $95 \%$, 94.9-100) (Table 2; Figure 6B).

\section{With the amended text}

All in all, the sensitivity rate for both markers was $93.3 \%$ (42/45, CI $95 \%, 81.7-98.6)$, with a specificity level of $100 \%$ (68/68, CI 95 \%, 94.7-100) (Table 2; Figure 6B).

2. (Page 8: End of "Validation and diagnostic accuracy of miR-203 and miR-205 expression in FNA samples")

Please replace

Moreover, negative predictive values were of $95.9 \%$ (95\% CI, 88.6-99.1\%) and positive predictive values of $100 \%$ (95 \% CI, 90.9-100 \%) for both microRNAs (Table 3).

\section{With the amended text}

Moreover, negative predictive values were of $95.9 \%$ (95\% CI, 88.6-99.1\%) and positive predictive values of $100 \%(95 \% \mathrm{CI}, 91.5-100 \%)$ for both microRNAs (Table 3).

* Correspondence: alopescarvalho@gmail.com; andre.vettore@gmail.com

${ }^{4}$ Department of Head and Neck Surgery, Barretos Cancer Hospital, Barretos, Brazil

${ }^{5}$ Laboratory of Cancer Molecular Biology, Department of Biological Sciences, Federal University of São Paulo, Diadema, Brazi

Full list of author information is available at the end of the article 
3. (Page 8: Table 2) - Please replace:

Table 2 Sensitivity and specificity values of microRNAs evaluated in discriminating metastatic and non-metastatic lymph nodes in FFPE and FNA biopsies from lymph node samples

\begin{tabular}{|c|c|c|c|c|c|c|}
\hline \multirow[t]{3}{*}{ microRNA } & \multirow{3}{*}{ Cutoff $^{\mathrm{a}}$} & \multicolumn{4}{|l|}{ Sensitivity } & \multirow{3}{*}{$\begin{array}{l}\text { Specificity } \\
\text { Non-metastatic } \\
\%(95 \% \text { Cl) (n) }\end{array}$} \\
\hline & & Metastatic ${ }^{\mathrm{b}}$ & Macrometastases & Micrometastases & Isolated tumor cells & \\
\hline & & $\%(95 \%$ Cl) (n) & $\%(95 \% C l)(n)$ & $\%(95 \%$ Cl) (n) & $\%(95 \%$ Cl) (n) & \\
\hline \multicolumn{7}{|c|}{ FFPE samples } \\
\hline miR-200a & 5.96 & 76.0 (54.5-89.8) (19/25) & $94.4(70.6-99.7)(17 / 18)$ & $40.0(7.3-82.9)(2 / 5)$ & $0(0-80.2)(0 / 2)$ & $100(71.7-100)(13 / 13)$ \\
\hline miR-200c & 2.33 & $88.0(67.7-96.8)(22 / 25)$ & $100(78.1-100)(17 / 18)$ & $80.0(29.9-98.9)(4 / 5)$ & $0(0-80.2)(0 / 2)$ & $100(71.7-100))(13 / 13)$ \\
\hline $\operatorname{miR}-203$ & 1.96 & $100(83.4-100)(25 / 25)$ & $100(78.1-100)(17 / 18)$ & $100(46.3-100)(5 / 5)$ & $100(19.8-100)(2 / 2)$ & $100(71.7-100)(13 / 13)$ \\
\hline $\operatorname{miR}-205$ & 1.54 & $100(83.4-100)(25 / 25)$ & $100(78.1-100)(17 / 18)$ & $100(46.3-100)(5 / 5)$ & $100(19.8-100)(2 / 2)$ & $100(71.7-100)(13 / 13)$ \\
\hline \multicolumn{7}{|c|}{ FNA samples classified by cytology } \\
\hline miR-203 & 10 & $100(91.5-100)(42 / 42)$ & $100(91.5-100)(42 / 42)$ & $\mathrm{N} / \mathrm{A}$ & N/A & $100(94.9-100)(71 / 71)$ \\
\hline miR-205 & 10 & $100(91.5-100)(42 / 42)$ & $100(91.5-100)(42 / 42)$ & N/A & N/A & $100(94.9-100)(71 / 71)$ \\
\hline \multicolumn{7}{|c|}{ FNA samples classified by histology } \\
\hline $\operatorname{miR}-203$ & 10 & $92.9(80.5-98.4)(68 / 71)$ & $100(89.3-100)(68 / 68)$ & $0(0)(0 / 2)$ & $0(0)(0 / 1)$ & $100(94.9-100)(71 / 71)$ \\
\hline miR-205 & 10 & $92.9(80.5-98.4)(68 / 71)$ & $100(89.3-100)(68 / 68)$ & $0(0)(0 / 2)$ & $0(0)(0 / 1)$ & $100(94.9-100)(71 / 71)$ \\
\hline
\end{tabular}

FFPE, formalin-fixed paraffin embedded; FNA, fine-needle aspiration; PPV, positive predictive value; NPV, negative predictive value; AUC, area under the ROC curve; $\mathrm{Cl}$, confidence interval; N/A, not applicable, FFPE, formalin-fixed paraffin embedded; FNA, fine-needle aspiration; PPV, positive predictive value; NPV, negative predictive value; AUC, area under the ROC curve; $\mathrm{Cl}$, confidence interval

${ }^{a}$ the cutoff values for FFPE samples were determined according to the Youden index (value in which the difference between sensitivity and 1-specificity is maximum) obtained from the ROC curves

${ }^{b}$ the "metastatic" group comprises all cases with positive lymph nodes (macrometastases, micrometastases or isolated tumor cells)

With the amended table:

Table 2 Sensitivity and specificity values of microRNAs evaluated in discriminating metastatic and non-metastatic lymph nodes in FFPE and FNA biopsies from lymph node samples

\begin{tabular}{|c|c|c|c|c|c|c|}
\hline \multirow[t]{3}{*}{ microRNA } & \multirow{3}{*}{ Cutoff $^{a}$} & \multicolumn{4}{|l|}{ Sensitivity } & \multirow{3}{*}{$\begin{array}{l}\text { Specificity } \\
\text { Non-metastatic } \\
\%(95 \% \text { Cl) (n) }\end{array}$} \\
\hline & & Metastatic $^{b}$ & Macrometastases & Micrometastases & Isolated tumor cells & \\
\hline & & $\%(95 \% C l)(n)$ & $\%(95 \% C l)(n)$ & $\%(95 \% \mathrm{Cl})(\mathrm{n})$ & $\%(95 \% C l)(n)$ & \\
\hline \multicolumn{7}{|c|}{ FFPE samples } \\
\hline miR-200a & 5.96 & $76.0(54.5-89.8)(19 / 25)$ & $94.4(70.6-99.7)(17 / 18)$ & $40.0(7.3-82.9)(2 / 5)$ & $0(0-80.2)(0 / 2)$ & $100(71.7-100)(13 / 13)$ \\
\hline miR-200c & 2.33 & $88.0(67.7-96.8)(22 / 25)$ & $100(78.1-100)(18 / 18)$ & $80.0(29.9-98.9)(4 / 5)$ & $0(0-80.2)(0 / 2)$ & $100(71.7-100))(13 / 13)$ \\
\hline miR-203 & 1.96 & $100(83.4-100)(25 / 25)$ & $100(78.1-100)(18 / 18)$ & $100(46.3-100)(5 / 5)$ & $100(19.8-100)(2 / 2)$ & $100(71.7-100)(13 / 13)$ \\
\hline miR-205 & 1.54 & $100(83.4-100)(25 / 25)$ & $100(78.1-100)(18 / 18)$ & $100(46.3-100)(5 / 5)$ & $100(19.8-100)(2 / 2)$ & $100(71.7-100)(13 / 13)$ \\
\hline \multicolumn{7}{|c|}{ FNA samples classified by cytology } \\
\hline miR-203 & 10 & $100(91.5-100)(42 / 42)$ & $100(91.5-100)(42 / 42)$ & N/A & N/A & $100(94.9-100)(71 / 71)$ \\
\hline miR-205 & 10 & $100(91.5-100)(42 / 42)$ & $100(91.5-100)(42 / 42)$ & N/A & N/A & $100(94.9-100)(71 / 71)$ \\
\hline \multicolumn{7}{|c|}{ FNA samples classified by histology } \\
\hline miR-203 & 10 & $93.3(81.7-98.6)(42 / 45)$ & $100(91.5-100)(42 / 42)$ & $0(0)(0 / 2)$ & $0(0)(0 / 1)$ & $100(94.7-100)(68 / 68)$ \\
\hline miR-205 & 10 & $93.3(81.7-98.6)(42 / 45)$ & $100(91.5-100)(42 / 42)$ & $0(0)(0 / 2)$ & $0(0)(0 / 1)$ & $100(94.7-100)(68 / 68)$ \\
\hline
\end{tabular}

FFPE, formalin-fixed paraffin embedded; FNA, fine-needle aspiration; PPV, positive predictive value; NPV, negative predictive value; AUC, area under the ROC curve; $\mathrm{Cl}$, confidence interval; N/A, not applicable, FFPE, formalin-fixed paraffin embedded; FNA, fine-needle aspiration; PPV, positive predictive value; NPV, negative predictive value; AUC, area under the ROC curve; $\mathrm{Cl}$, confidence interval

${ }^{a}$ the cutoff values for FFPE samples were determined according to the Youden index (value in which the difference between sensitivity and 1-specificity is maximum) obtained from the ROC curves

${ }^{b}$ the "metastatic" group comprises all cases with positive lymph nodes (macrometastases, micrometastases or isolated tumor cells) 
4. (Page 10: Table 3) - Please replace:

Table 3 Accuracy characteristics of microRNAs in discriminating metastatic and non-metastatic lymph nodes in FFPE and FNA biopsies from lymph node samples

\begin{tabular}{|c|c|c|c|c|}
\hline \multirow[t]{2}{*}{ microRNA } & PPV & NPV & Accuracy & \multirow[t]{2}{*}{ AUC (95 \% Cl) } \\
\hline & $\%(95 \% \mathrm{Cl})$ & $\%(95 \% \mathrm{Cl})$ & $\%(95 \% \mathrm{Cl})$ & \\
\hline \multicolumn{5}{|c|}{ FFPE samples } \\
\hline miR-200a & $100(82.2-100.0)$ & $68.4(43.5-87.3)$ & $84.2(68.1-93.4)$ & $0.92(0.83-0.99)$ \\
\hline miR-200c & $100(84.4-100.0)$ & $81.2(54.34-95.73)$ & $92.1(77.5-97.9)$ & $0.94(0.85-1.0)$ \\
\hline miR-203 & $100(86.2-100)$ & $100(75.1-100)$ & $100(88.6-100)$ & $1.0(0-1.0)$ \\
\hline $\operatorname{miR}-205$ & $100(86.2-100)$ & $100(75.1-100)$ & $100(88.6-100)$ & $1.0(0-1.0)$ \\
\hline \multicolumn{5}{|c|}{ FNA samples classified by cytology } \\
\hline miR-203 & $100(91.5-100)$ & $100(94.9-100)$ & $100(96.05-100)$ & $1.0(0-1.0)$ \\
\hline miR-205 & $100(91.5-100)$ & $100(94.9-100)$ & $100(96.05-100)$ & $1.0(0-1.0)$ \\
\hline \multicolumn{5}{|c|}{ FNA samples classified by histology } \\
\hline miR-203 & $100(90.9-100)$ & $95.9(88.6-99.1)$ & $97.3(92.1-99.4)$ & $0.963(0.921-1.0)$ \\
\hline miR-205 & $100(93.2-100)$ & $94.6(85.1-98.8)$ & $96.7(93.1-100)$ & $0.966(0.921-1.0)$ \\
\hline
\end{tabular}

FFPE, formalin-fixed paraffin embedded; FNA, fine-needle aspiration; PPV, positive predictive value; NPV, negative predictive value; AUC, area under the ROC curve; $\mathrm{Cl}$, confidence interval

With the amended table:

Table 3 Accuracy characteristics of microRNAs in discriminating metastatic and non-metastatic lymph nodes in FFPE and FNA biopsies from lymph node samples

\begin{tabular}{|c|c|c|c|c|}
\hline \multirow[t]{2}{*}{ microRNA } & PPV & NPV & Accuracy & \multirow[t]{2}{*}{ AUC (95 \% Cl) } \\
\hline & $\%(95 \% \mathrm{Cl})$ & $\%(95 \% \mathrm{Cl})$ & $\%(95 \% \mathrm{Cl})$ & \\
\hline \multicolumn{5}{|c|}{ FFPE samples } \\
\hline miR-200a & $100(82.2-100.0)$ & $68.4(43.5-87.3)$ & $84.2(68.1-93.4)$ & $0.92(0.83-0.99)$ \\
\hline $\operatorname{miR}-200 c$ & $100(84.4-100.0)$ & $81.2(54.34-95.73)$ & $92.1(77.5-97.9)$ & $0.94(0.85-1.0)$ \\
\hline miR-203 & $100(86.2-100)$ & $100(75.1-100)$ & $100(88.6-100)$ & $1.0(0-1.0)$ \\
\hline miR-205 & $100(86.2-100)$ & $100(75.1-100)$ & $100(88.6-100)$ & $1.0(0-1.0)$ \\
\hline \multicolumn{5}{|c|}{ FNA samples classified by cytology } \\
\hline miR-203 & $100(91.5-100)$ & $100(94.9-100)$ & $100(96.05-100)$ & $1.0(0-1.0)$ \\
\hline miR-205 & $100(91.5-100)$ & $100(94.9-100)$ & $100(96.05-100)$ & $1.0(0-1.0)$ \\
\hline \multicolumn{5}{|c|}{ FNA samples classified by histology } \\
\hline miR-203 & $100(91.5-100)$ & $95.9(88.6-99.1)$ & $97.3(92.1-99.4)$ & $0.963(0.921-1.0)$ \\
\hline miR-205 & $100(91.5-100)$ & 95.9 (88.6-99.1) & 97.3 (92.1-99.4) & $0.966(0.921-1.0)$ \\
\hline
\end{tabular}

FFPE, formalin-fixed paraffin embedded; FNA, fine-needle aspiration; PPV, positive predictive value; NPV, negative predictive value; AUC, area under the ROC curve; $\mathrm{Cl}$, confidence interval 


\section{Author details}

${ }^{1}$ Laboratory of Cancer Molecular Biology, Department of Biological Sciences, Federal University of São Paulo, Diadema, Brazil. ²Department of Pathology, Barretos Cancer Hospital, Barretos, Brazil. ${ }^{3}$ Molecular Oncology Research

Center, Barretos Cancer Hospital, Barretos, Brazil. ${ }^{4}$ Department of Head and Neck Surgery, Barretos Cancer Hospital, Barretos, Brazil. ${ }^{5}$ Laboratory of Cancer Molecular Biology, Department of Biological Sciences, Federal University of São Paulo, Diadema, Brazil. ${ }^{6}$ Cancer and Stem Cell Biology Program,

Duke-NUS Graduate Medical School, Singapore, Singapore. ${ }^{7}$ Departamento de Cirurgia de Cabeça e Pescoço, Hospital de Câncer de Barretos, Rua Antenor Duarte Villela, 1331, Barretos, SP 14784-400, Brazil. ' Laboratório de Biologia Molecular do Câncer, UNIFESP, Rua Pedro de Toledo, $669-11^{\circ}$ andar, L11B, São Paulo, SP 04039-032, Brazil.

Received: 18 June 2015 Accepted: 18 June 2015

Published online: 01 July 2015

\section{Reference}

1. de Carvalho AC, Scapulatempo-Neto C, Maia DC, Evangelista AF, Morini MA, Carvalho AL, et al. Accuracy of microRNAs as markers for the detection of neck lymph node metastases in patients with head and neck squamous cell carcinoma. BMC Med. 2015;13(1):108. doi:10.1186/s12916-015-0350-3.

\section{Submit your next manuscript to BioMed Central and take full advantage of:}

- Convenient online submission

- Thorough peer review

- No space constraints or color figure charges

- Immediate publication on acceptance

- Inclusion in PubMed, CAS, Scopus and Google Scholar

- Research which is freely available for redistribution 